\title{
BMJ Open Using multimedia tools and high- fidelity simulations to improve medical students' resuscitation performance: an observational study
}

\author{
Candice Wang, ${ }^{1,2}$ Chin-Chou Huang, ${ }^{1,3,4,5}$ Shing-Jong Lin, ${ }^{3,4,6,7}$ \\ Jaw-Wen Chen $3,4,5,6$
}

To cite: Wang C, Huang C-C, Lin S-J, et al. Using multimedia tools and highfidelity simulations to improve medical students' resuscitation performance: an observational study. BMJ Open 2016;6:e012195. doi:10.1136/bmjopen-2016012195

- Prepublication history and additional material is available. To view please visit the journal (http://dx.doi.org/ 10.1136/bmjopen-2016012195).

Received 7 April 2016 Revised 3 September 2016 Accepted 7 September 2016

CrossMark

For numbered affiliations see end of article.

Correspondence to Dr Chin-Chou Huang; cchuang4@vghtpe.gov.tw

\section{ABSTRACT}

Objectives: The goal of our study was to shed light on educational methods to strengthen medical students' cardiopulmonary resuscitation (CPR) leadership and team skills in order to optimise CPR understanding and success using didactic videos and high-fidelity simulations.

Design: An observational study.

Setting: A tertiary medical centre in Northern Taiwan. Participants: A total of 104 5-7th year medical students, including 72 men and 32 women.

Interventions: We provided the medical students with a 2-hour training session on advanced CPR. During each class, we divided the students into 1-2 groups; each group consisted of 4-6 team members. Medical student teams were trained by using either method A or B. Method A started with an instructional CPR video followed by a first CPR simulation. Method $B$ started with a first CPR simulation followed by an instructional CPR video. All students then participated in a second CPR simulation.

Outcome measures: Student teams were assessed with checklist rating scores in leadership, teamwork and team member skills, global rating scores by an attending physician and video-recording evaluation by 2 independent individuals.

Results: The 104 medical students were divided into 22 teams. We trained 11 teams using method $A$ and 11 using method B. Total second CPR simulation scores were significantly higher than first CPR simulation scores in leadership $(p<0.001)$, teamwork $(p<0.001)$ and team member skills $(p<0.001)$. For methods A and B students' first CPR simulation scores were similar, but method $A$ students' second CPR simulation scores were significantly higher than those of method $B$ in leadership skills $(p=0.034)$, specifically in the support subcategory $(p=0.049)$.

Conclusions: Although both teaching strategies improved leadership, teamwork and team member performance, video exposure followed by CPR simulation further increased students' leadership skills compared with CPR simulation followed by video exposure.
Strengths and limitations of this study

- Although the study was designed only observationally, the baseline characteristics of the participants in the two method groups were similar, including gender, degree, experience on resuscitation and student numbers in each team.

- Owing to the ethical prioritisation of student learning experiences, all student groups received both a first cardiopulmonary resuscitation (CPR) simulation and video showing, though in varying chronological orders.

- The allocation of the students to two methods was random; student groups were trained by video exposure followed by a first CPR simulation (method A) during even numbered months, and by a first CPR simulation followed by video exposure (method B) during odd numbered months.

- The evaluation process included real-time evaluation by one attending physician who taught the students, and video-recording evaluation by two independent individuals.

\section{INTRODUCTION}

Cardiopulmonary resuscitation (CPR) is one of the most important procedures to treat cardiac arrest. $^{1}{ }^{2}$ In a chart review of advanced cardiac life support (ACLS) protocol execution in patients with cardiac arrest, adherence to ACLS protocols was correlated with increased return of spontaneous circulation. ${ }^{3}$ Significant interaction has also been noted between the quality of CPR provided, intervention and survival to hospital discharge. ${ }^{4}$ However, the quality of CPR often does not meet published guideline recommendations, even when performed by welltrained hospital staff. ${ }^{5}$ The Institute of Medicine urges hospitals to actively seek improvement of CPR performance by implementing "continuous quality improvement 
programs...tracking system performance... and ensuring that personnel are trained to respond competently to cardiac arrest". ${ }^{6}$ This indicates that a variety of didactic methods may be needed to improve the teaching strategy of CPR training.

Leadership and teamwork skills are important components of $\mathrm{CPR},{ }^{7-14}$ and these concepts have been implemented in both the European Resuscitation Council's CPR guidelines ${ }^{12}$ and the American Heart Association (AHA) guidelines for CPR and Emergency Cardiovascular Care. ${ }^{13}$ To effectively teach these skills, high-fidelity simulations offer a safe, low-risk environment with minimal confounding variables. ${ }^{15}$ This gives students the opportunity to practice procedures and team/leadership skills in a controlled setting where evaluators may focus on leadership and teamwork skills. ${ }^{14}$

Multimedia tools for learning CPR skills in medical education have shown to be beneficial. One such study showed that a brief CPR video training resulted in improved CPR quality and responsiveness among high school students. ${ }^{16}$ In another study comparing traditional lectures for teaching resuscitation scenarios versus video-based instructions versus low-fidelity or high-fidelity simulation activities, students with videobased or simulation-based training displayed greater skill performance. ${ }^{17}$ This suggests that training with video exposure may have a positive effect on CPR performance. It is thus important to consider the joint effects of training video exposure and high-fidelity simulations.

The goal of our study was to investigate the joint effects of multimedia tools and high-fidelity simulations with varying chronological orders. The findings of our study will help us shed light on educational methods to strengthen medical students' CPR leadership and team skills in order to optimise CPR understanding and success.
METHODS

\section{Design}

We provided a 2-hour training session on advanced CPR for 5-7th year medical students. During each session, we divided students into 1-2 groups; each group consisted of 4-6 team members. Across 13 months, we trained student groups with either method A or B. During even numbered months, student groups were trained with method A. During odd numbered months, student groups were trained with method B. Method A began with the showing of an instructional resuscitation video stressing on the importance of leadership, teamwork and team member skills. Following the video, we gave students a first CPR simulation. Next, class discussion with an attending physician highlighted the strengths and weaknesses of this first CPR simulation performance. Finally, we gave the students a second CPR simulation, in which a CPR simulation was practised again. Method B began with a first CPR simulation and subsequent discussion. Next, we showed the resuscitation video, and ended the training with a second CPR simulation (figure 1).

In the CPR simulations, there were four different scenarios of in-hospital cardiac arrest. The CPR procedures were similar because all four scenarios included ventricular fibrillation/ventricular tachycardia and pulseless electrical activity/asystole. The simulations were practised using a high-fidelity simulator (Simman, Laerdal Medical Corporation).

\section{Participants}

We recruited 5th-7th year medical students to participate in our advanced CPR training programme at Taipei Veterans General Hospital, Taipei, Taiwan from December 2012 to December 2013.
Figure 1 Flow chart of the study.

\section{4 medical students (20 teams with 4-6 students)}

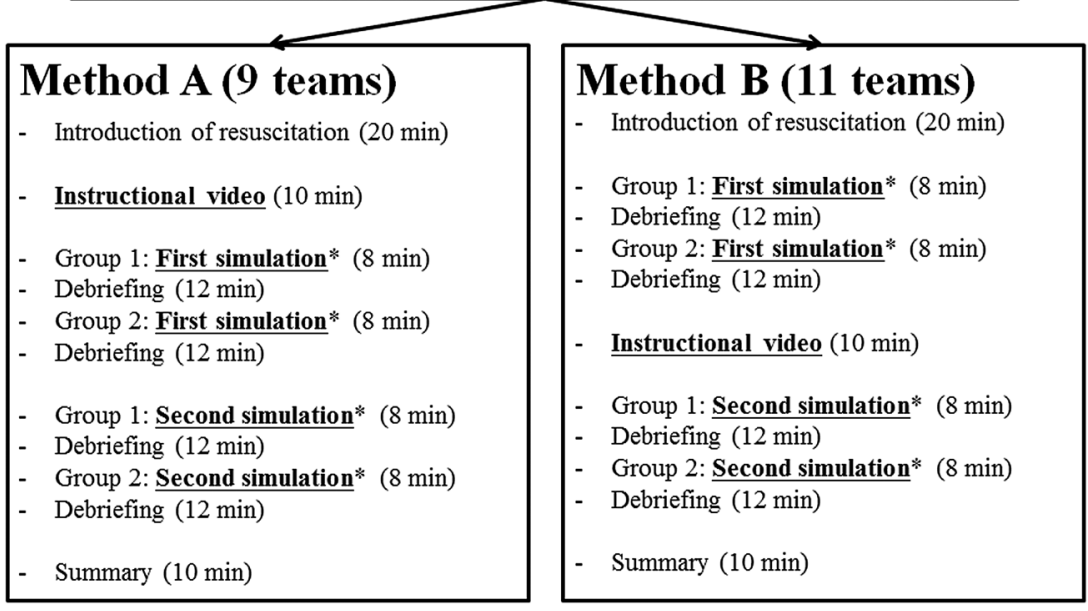

*Evaluation processes including real-time evaluation and vide-recording evaluation: 1. Real-time evaluation by one attending physician using a formal evaluation form.

2. Video-recording evaluation by two independent individuals to record the reaction times 
The study protocol was exempt from review by the ethics committee of Taipei Veterans General Hospital. All results have been reported in an anonymous fashion and pose minimal risk to the students of the study. One research assistant at the institute conducted data collection and coding.

\section{Instructional video}

The didactic video was chapter 4, 'Megacode and Team Resuscitation Concept' from the AHA's 2005 ACLS DVD course. It began with a descriptive overview of team dynamics, team leader traits and team member traits. Elements of team dynamics included closed-loop communication, clear messages, clear roles and responsibilities, knowing one's limitations, knowledge sharing, constructive intervention, re-evaluation and summarising, and mutual respect. Team leader traits included organising the group and assign team roles, back up team members, model excellent team behaviour, train and coach, and facilitate understanding. Team member traits included preparedness, well-practised, proficient in algorithm knowledge and committed to success. Next, a resuscitation team performed an exemplary CPR simulation to demonstrate the correct execution of the resuscitation protocol, leadership responsibilities and team member responsibilities. The video then concluded with a reiteration of the elements of team dynamics.

\section{Real-time evaluation}

Evaluation processes for the first and second CPR simulation were the same. An attending physician graded medical student teams from the observation room as they performed CPR simulations. He assessed each team as one unit using a formal evaluation form that included checklist rating scores and global rating scores.

The checklist rating form was comprised of 20 items in three categories: leadership, teamwork and team member skills. The checklist rating scores ranged from 1 to 5 for each item. A score of 1 was the lowest score and a score of 5 was the highest score.

The leadership category consisted of four subcategories: (1) organisation, which meant the ability to designate roles to team members such as chest compression, oxygen delivery, defibrillation, intravenous (IV) medication and recording; (2) order giving, which represented the ability to give orders to team members, communicating the necessary timing and sequence of their actions; (3) support, characterised by the ability to aid team members in their various roles; (4) awareness, which described the ability to perceive the situation and understand what actions must be taken.

The teamwork category consisted of six subcategories: (1) communication, which meant the ability to effectively communicate with and deliver clear, concise messages to teammates; (2) cooperation, represented by the ability to cohesively work with teammates to achieve a common goal using patience, understanding and respect; (3) experience-sharing, characterised by the ability to share the simulation experience with fellow teammates; (4) ordering, which described the ability to effectively give orders to teammates; (5) ACLS principle adherence, which meant the ability to follow ACLS guidelines and protocols; and (6) task completion, signified by the ability to successfully complete the simulation.

The team member category consisted of 10 subcategories: (1) patient contact, which meant the ability to make initial contact with the patient to determine consciousness and state of mind; (2) chest compression, which represented the ability to perform effective chest compressions on a patient; (3) airway check, characterised by the ability to check patient's airways for obstructions; (4) oxygen delivery, which described the ability to consistently deliver oxygen to the patient using a bag valve mask; (5) defibrillation, which meant the ability to set up and use a defibrillator; (6) intubation, represented by the ability to efficiently and successfully intubate the patient; (7) IV medication, signified by the ability to inject appropriate medication through the patient's IV; (8) monitoring, which represented the ability to monitor patient's vital signs; (9) ECG recognition, characterised by the ability to monitor and understand patient ECG outputs; and (10) recording, which meant the ability to accurately record events of the simulation, including times for each of the protocol steps, and procedures or medications used.

On the global rating form, the attending physician gave students an overall performance rating on a scale of $1-5 ; 1$ was considered poor; 2 , fair; 3 , good; 4 , very good and 5, excellent.

The evaluation form is an observational tool. The content validity was established by a cardiologist, an emergency physician and a nursing specialist at our institute (see online supplementary appendix 1 ).

\section{Video-recording evaluation}

Two independent individuals blinded to the study watched the students' first and second CPR simulation video recordings to record reaction times for each of the following chronological CPR procedures: patient contact, chest compression and defibrillation. If procedural steps were carried out, reaction times were recorded and those steps were considered complete. If steps were not performed during the stimulation, no reaction time was recorded and those steps were considered incomplete.

\section{Data processing and analysis}

We recorded all of the examinees' evaluation results inclusively and compared them statistically using SPSS V.15.0 software (SPSS, Chicago, Illinois, USA). We compared the baseline characteristics of the participants in method A and B groups. Categorical variables were analysed using $\chi^{2}$ tests or Fisher's exact tests, including gender, degree and experience on resuscitation. Student numbers in each team between method A and B groups were compared using the Student's t-test. 
Each checklist rating score and global rating score was expressed as mean (SD). We performed a paired t-test to compare the first simulation and second simulation scores in all groups, method A and B groups. We performed the Student's t-test to compare the checklist rating scores and global rating scores of the first and second CPR simulation in method A and B groups.

We performed Pearson's $\chi^{2}$ analysis to compare the completion frequencies of each CPR procedural step between different groups. We subtracted times recorded for each action and calculated this difference in seconds. Reaction times of chest compressions were defined as the time from discovery of unconsciousness until chest compressions began. Reaction times of defibrillation were defined as the time from discovery of unconsciousness until shock was delivered. ${ }^{18}$ We performed a paired t-test to compare the reaction times of first simulation and second simulation scores in all groups, method A and B groups. We performed the Student's t-test to compare the reaction times of the first CPR simulation and second CPR simulation, and the differences in method A and B groups. We inferred statistical significance based on a two-sided $p$ value of $<0.05$.

\section{RESULTS}

\section{Participants}

We enrolled 104 medical students (72 men and 32 women) in the study, including 33 seventh year medical students, 14 sixth year medical students and 57 fifth year medical students. Sixty-three $(60.6 \%)$ students had previous resuscitation experience. We divided all students into 22 teams. We then trained 11 teams using method A and 11 teams using method B. A total of 27 students (54.0\%) in method A group and 36 students $(66.7 \%)$ in method B group had previous resuscitation experience $(p=0.187)$. There were no differences in the baseline characteristics of the participants trained by each method. The mean student numbers in each team of the two methods were also similar $(\operatorname{method} \mathrm{A}$ vs $\mathrm{B}=4.5$ \pm 0.5 vs $4.9 \pm 0.8, \mathrm{p}=0.236$; table 1 ). Each student group completed first and second CPR simulations during the 2-hour training session.

\section{Comparing the first and second CPR simulation scores in all groups}

The overall second CPR simulation scores were significantly higher than first CPR simulation scores in three categories: leadership $(p<0.001)$, teamwork $(p<0.001)$ and team member skills $(\mathrm{p}<0.001)$. Within the leadership category, the following subcategories showed second CPR simulation scores that were significantly higher than first CPR simulation scores: organisation $(\mathrm{p}=0.010)$, giving orders $(\mathrm{p}=0.002)$, support $(\mathrm{p}=0.001)$ and awareness $(\mathrm{p}=0.001)$. Within the teamwork category, the following subcategories showed second CPR simulation scores that were significantly higher than first CPR simulation scores: communication $(\mathrm{p}=0.003)$, cooperation
Table 1 Comparing the baseline characteristics in method $A$ and $B$ groups

\begin{tabular}{llll}
\hline & $\begin{array}{l}\text { Method A } \\
(\mathbf{n}=11)\end{array}$ & $\begin{array}{l}\text { Method B } \\
(\mathbf{n = 1 1 )}\end{array}$ & p Value \\
\hline $\begin{array}{l}\text { Participants } \\
\text { Gender }\end{array}$ & 50 & 54 & \\
$\quad$ Male & $32(64.0 \%)$ & $40(74.1 \%)$ & \\
$\quad$ Female & $18(36.0 \%)$ & $14(25.9 \%)$ & \\
Degree & & & 0.852 \\
$\quad$ Year 7 & $17(34.0 \%)$ & $16(29.6 \%)$ & \\
$\quad$ Year 6 & $6(12.0 \%)$ & $8(14.8 \%)$ & \\
$\quad$ Year 5 & $27(54.0 \%)$ & $30(55.6 \%)$ & \\
Experience on resuscitation & & 0.187 \\
$\quad$ Yes & $27(54.0 \%)$ & $36(66.7 \%)$ & \\
$\quad$ No & $23(46.0 \%)$ & $18(33.3 \%)$ & \\
Student numbers & $4.5 \pm 0.5$ & $4.9 \pm 0.8$ & 0.236 \\
in each team & & & \\
\hline
\end{tabular}

( $p<0.001)$, sharing the experience $(\mathrm{p}=0.002)$, ordering $(\mathrm{p}<0.001)$, following ACLS principles $(\mathrm{p}<0.001)$ and completion $(\mathrm{p}=0.004)$. Within the team member category, the following subcategories showed second CPR simulation scores that were significantly higher than first CPR simulation scores: contact patient $(\mathrm{p}=0.003)$, chest compression $(\mathrm{p}<0.001)$ and IV medication $(\mathrm{p}<0.001)$. The global rating scores of the second CPR simulation scores were also significantly higher than the first CPR simulation scores $(p<0.001$; table 2$)$.

In method $\mathrm{A}$, students scored significantly higher on their second CPR simulation than first CPR simulation in three categories: leadership $(p<0.001)$, teamwork $(p<0.001)$ and team member skills $(p<0.001)$. The global rating scores of the second CPR simulation scores were also significantly higher than the first CPR simulation scores $(\mathrm{p}=0.011$; table 3$)$.

In method B, students scored significantly higher on their second CPR simulation than the first CPR simulation in three categories: leadership $(p<0.001)$, teamwork $(p<0.001)$ and team member skills $(p<0.001)$. The global rating scores of the second CPR simulation scores were also significantly higher than the first CPR simulation scores $(\mathrm{p}=0.001$; table 4$)$.

\section{Comparing the first CPR simulation scores of methods A and $B$}

The first CPR simulation scores in leadership, teamwork, team member skills and global rating scores did not differ significantly between students taught by method A versus B (table 5).

\section{Comparing the second CPR simulation scores of methods $A$ and $B$}

Method A second CPR simulation scores in leadership were significantly higher than method $\mathrm{B}$ second CPR simulation scores in leadership ( $\mathrm{p}=0.034$; table 5$)$. Within the leadership category, the support subcategory score was significantly higher for students taught using method 
Table 2 Comparing first simulation and second simulation scores in all groups $(n=22)$

\begin{tabular}{|c|c|c|c|c|}
\hline Scoring category & Subcategory & First simulation & Second simulation & p Value \\
\hline \multirow[t]{5}{*}{ Leadership } & Overall & $2.5 \pm 0.7$ & $3.2 \pm 0.6$ & $<0.001$ \\
\hline & 1. Organisation & $2.6 \pm 0.8$ & $3.2 \pm 0.6$ & 0.010 \\
\hline & 2. Giving orders & $2.6 \pm 0.8$ & $3.3 \pm 0.6$ & 0.002 \\
\hline & 3. Support & $2.4 \pm 0.6$ & $3.2 \pm 0.7$ & 0.001 \\
\hline & 4. Awareness & $2.5 \pm 0.6$ & $3.3 \pm 0.6$ & $<0.001$ \\
\hline \multirow[t]{7}{*}{ Teamwork } & Overall & $2.7 \pm 0.6$ & $3.4 \pm 0.6$ & $<0.001$ \\
\hline & 1. Communication & $2.9 \pm 0.5$ & $3.4 \pm 0.6$ & 0.003 \\
\hline & 2. Cooperation & $2.9 \pm 0.6$ & $3.7 \pm 0.5$ & $<0.001$ \\
\hline & 3. Sharing the experience & $2.9 \pm 0.5$ & $3.4 \pm 0.5$ & 0.002 \\
\hline & 4. Ordering & $2.6 \pm 0.7$ & $3.4 \pm 0.5$ & $<0.001$ \\
\hline & 5. Follow ACLS principles & $2.6 \pm 0.8$ & $3.2 \pm 0.6$ & 0.007 \\
\hline & 6. Completion & $2.7 \pm 0.5$ & $3.2 \pm 0.7$ & 0.004 \\
\hline \multirow[t]{11}{*}{ Team member } & Overall & $2.7 \pm 0.7$ & $3.1 \pm 0.6$ & $<0.001$ \\
\hline & 1. Contact patient & $2.8 \pm 0.6$ & $3.3 \pm 0.5$ & 0.003 \\
\hline & 2. Chest compression & $2.4 \pm 0.7$ & $3.2 \pm 0.6$ & $<0.001$ \\
\hline & 3. Check airway & $2.7 \pm 0.5$ & $2.9 \pm 0.6$ & 0.203 \\
\hline & 4. Give $\mathrm{O}_{2}$ & $2.8 \pm 0.6$ & $3.2 \pm 0.5$ & 0.055 \\
\hline & 5. Defibrillation & $2.6 \pm 1.0$ & $3.1 \pm 0.7$ & 0.119 \\
\hline & 6. Intubation & $2.6 \pm 0.7$ & $2.8 \pm 1.0$ & 0.657 \\
\hline & 7. IV medication & $2.5 \pm 0.8$ & $3.4 \pm 0.6$ & $<0.001$ \\
\hline & 8. Monitoring & $3.0 \pm 0.6$ & $3.2 \pm 0.4$ & 0.201 \\
\hline & 9. ECG recognition & $3.2 \pm 0.7$ & $3.3 \pm 0.5$ & 0.346 \\
\hline & 10. Recording & $2.4 \pm 0.8$ & $2.8 \pm 0.7$ & 0.093 \\
\hline Global rating & & $2.7 \pm 0.5$ & $3.4 \pm 0.5$ & $<0.001$ \\
\hline
\end{tabular}

ACLS, advanced cardiopulmonary life support; IV, intravenous.

Table 3 Comparing first simulation and second simulation scores in method A groups $(n=11)$

\begin{tabular}{|c|c|c|c|c|}
\hline Scoring category & Subcategory & First simulation & Second simulation & p Value \\
\hline \multirow[t]{5}{*}{ Leadership } & Overall & $2.6 \pm 0.6$ & $3.4 \pm 0.5$ & $<0.001$ \\
\hline & 1. Organisation & $2.8 \pm 0.8$ & $3.4 \pm 0.5$ & 0.095 \\
\hline & 2. Giving orders & $2.8 \pm 0.7$ & $3.4 \pm 0.5$ & 0.054 \\
\hline & 3. Support & $2.6 \pm 0.5$ & $3.6 \pm 0.5$ & 0.002 \\
\hline & 4. Awareness & $2.4 \pm 0.5$ & $3.3 \pm 0.5$ & 0.006 \\
\hline \multirow[t]{7}{*}{ Teamwork } & Overall & $2.8 \pm 0.6$ & $3.3 \pm 0.5$ & $<0.001$ \\
\hline & 1. Communication & $3.1 \pm 0.3$ & $3.4 \pm 0.5$ & 0.166 \\
\hline & 2. Cooperation & $3.0 \pm 0.5$ & $3.7 \pm 0.5$ & 0.012 \\
\hline & 3. Sharing the experience & $2.9 \pm 0.6$ & $3.1 \pm 0.4$ & 0.346 \\
\hline & 4. Ordering & $2.4 \pm 0.7$ & $3.3 \pm 0.5$ & 0.020 \\
\hline & 5. Follow ACLS principles & $2.7 \pm 0.9$ & $3.1 \pm 0.4$ & 0.198 \\
\hline & 6. Completion & $2.7 \pm 0.5$ & $3.3 \pm 0.5$ & 0.026 \\
\hline \multirow[t]{11}{*}{ Team member } & Overall & $2.6 \pm 0.7$ & $3.1 \pm 0.7$ & $<0.001$ \\
\hline & 1. Contact patient & $2.6 \pm 0.5$ & $3.1 \pm 0.4$ & 0.026 \\
\hline & 2. Chest compression & $2.1 \pm 0.6$ & $3.4 \pm 0.5$ & $<0.001$ \\
\hline & 3. Check airway & $2.7 \pm 0.5$ & $3.0 \pm 0.6$ & 0.275 \\
\hline & 4. Give $\mathrm{O}_{2}$ & $2.9 \pm 0.6$ & $3.0 \pm 0.6$ & 0.715 \\
\hline & 5. Defibrillation & $2.6 \pm 1.0$ & $3.3 \pm 0.8$ & 0.135 \\
\hline & 6. Intubation & $2.7 \pm 0.6$ & $2.3 \pm 1.3$ & 0.623 \\
\hline & 7. IV medication & $2.6 \pm 0.7$ & $3.6 \pm 0.5$ & 0.008 \\
\hline & 8. Monitoring & $2.9 \pm 0.8$ & $3.1 \pm 0.4$ & 0.445 \\
\hline & 9. ECG recognition & $3.1 \pm 0.6$ & $3.3 \pm 0.5$ & 0.543 \\
\hline & 10. Recording & $2.2 \pm 0.8$ & $2.6 \pm 0.9$ & 0.443 \\
\hline Global rating & & $2.7 \pm 0.5$ & $3.4 \pm 0.5$ & 0.011 \\
\hline
\end{tabular}


Table 4 Comparing first simulation and second simulation scores in method B groups $(n=11)$

\begin{tabular}{llllr}
\hline Scoring category & Subcategory & First simulation & Second simulation & p Value \\
\hline Leadership & Overall & $2.4 \pm 0.7$ & $3.1 \pm 0.7$ & $<0.001$ \\
& 1. Organisation & $2.4 \pm 0.7$ & $3.0 \pm 0.6$ & 0.033 \\
& 2. Giving orders & $2.5 \pm 0.8$ & $3.3 \pm 0.6$ & 0.017 \\
3. Support & $2.3 \pm 0.6$ & $2.9 \pm 0.7$ & 0.039 \\
Teamwork & 4. Awareness & $2.5 \pm 0.7$ & $3.3 \pm 0.6$ & 0.009 \\
& Overall & $2.7 \pm 0.6$ & $3.4 \pm 0.6$ & $<0.001$ \\
& 1. Communication & $2.7 \pm 0.5$ & $3.5 \pm 0.7$ & 0.009 \\
& 2. Cooperation & $2.7 \pm 0.6$ & $3.7 \pm 0.5$ & $<0.001$ \\
3. Sharing the experience & $2.8 \pm 0.4$ & $3.5 \pm 0.5$ & 0.002 \\
& 4. Ordering & $2.7 \pm 0.6$ & $3.5 \pm 0.5$ & 0.009 \\
Team member & 5. Follow ACLS principles & $2.5 \pm 0.7$ & $3.3 \pm 0.6$ & 0.019 \\
& 6. Completion & $2.6 \pm 0.5$ & $3.2 \pm 0.8$ & 0.059 \\
& Overall & $2.7 \pm 0.7$ & $3.1 \pm 0.6$ & $<0.001$ \\
& 1. Contact patient & $2.9 \pm 0.5$ & $3.4 \pm 0.5$ & 0.055 \\
& 2. Chest compression & $2.5 \pm 0.7$ & $3.1 \pm 0.5$ & 0.052 \\
& 3. Check airway & $2.6 \pm 0.5$ & $2.8 \pm 0.6$ & 0.452 \\
& 4. Give O & $3.3 \pm 0.5$ & 0.035 \\
& 5. Defibrillation & $2.7 \pm 0.6$ & $2.9 \pm 0.7$ & 0.475 \\
& 6. Intubation & $2.6 \pm 1.0$ & $3.0 \pm 0.9$ & 0.325 \\
& 7. IV medication & $2.6 \pm 0.8$ & $3.4 \pm 0.7$ & 0.017 \\
8. Monitoring & $2.5 \pm 0.9$ & $3.2 \pm 0.4$ & 0.329 \\
9. ECG recognition & $3.0 \pm 0.4$ & $3.4 \pm 0.5$ & 0.513 \\
& 10. Recording & $3.2 \pm 0.8$ & $2.9 \pm 0.5$ & 0.199 \\
Global rating & & $2.5 \pm 0.8$ & $3.5 \pm 0.5$ & 0.001 \\
\hline
\end{tabular}

ACLS, advanced cardiopulmonary life support; IV, intravenous.

Table 5 Comparing first simulation scores and second simulation scores in method $A$ and $B$ groups $(n=11, n=11)$

\begin{tabular}{llll}
\hline Scoring category & $\begin{array}{l}\text { Method A } \\
(\mathbf{n}=\mathbf{1 1})\end{array}$ & $\begin{array}{l}\text { Method B } \\
(\mathbf{n}=\mathbf{1 1})\end{array}$ & $\mathbf{p ~ V a l u e}$ \\
\hline $\begin{array}{l}\text { First simulation } \\
\quad \text { Leadership }\end{array}$ & $2.6 \pm 0.6$ & $2.4 \pm 0.7$ & 0.096 \\
Teamwork & $2.8 \pm 0.6$ & $2.7 \pm 0.6$ & 0.359 \\
Team member & $2.6 \pm 0.7$ & $2.7 \pm 0.7$ & 0.331 \\
$\quad$ Global rating & $2.7 \pm 0.5$ & $2.6 \pm 0.5$ & 0.895 \\
Second simulation & & & \\
Leadership & $3.4 \pm 0.5$ & $3.1 \pm 0.7$ & 0.034 \\
Teamwork & $3.1 \pm 0.7$ & $3.1 \pm 0.6$ & 0.963 \\
Team member & $3.3 \pm 0.5$ & $3.4 \pm 0.6$ & 0.342 \\
Global rating & $3.4 \pm 0.5$ & $3.5 \pm 0.5$ & 0.920 \\
\hline
\end{tabular}

A than those taught using method B (method A vs $\mathrm{B}=3.6$ \pm 0.5 vs $2.9 \pm 0.7, p=0.049$ ). However, method A second CPR simulation scores in teamwork, team member skills and global rating scores were not significantly different than method B second CPR simulation scores.

\section{Comparing the completion frequencies and reaction times of each procedural step of methods A and B}

The completion frequencies of method $\mathrm{A}$ and $\mathrm{B}$ groups were $100 \%$ in the first and second simulations. All student teams performed patient contact, chest compression and defibrillation.

The reaction times recorded by two independent individuals were consistent. The mean reaction times of chest compression were $37.8 \pm 38.8$ and $40.1 \pm 39.6 \mathrm{~s}$, respectively $(\mathrm{p}=0.118)$. The mean reaction times of defibrillation were $179.6 \pm 78.4$ and $182.5 \pm 77.0 \mathrm{~s}$, respectively $(\mathrm{p}=0.057)$.

Overall, the chest compression reaction times of the second simulations were significantly shorter than those of the first simulations (first simulation vs second simulation $=43.3 \pm 38.8$ vs $25.1 \pm 16.4 \mathrm{~s}, \mathrm{p}=0.020$ ). Among method A groups, the chest compression reaction times of the second simulations was significantly shorter than those of the first simulations (first simulation vs second simulation $=64.6 \pm 53.6$ vs $23.3 \pm 8.9 \mathrm{~s}, \mathrm{p}=0.019)$. Among method B groups, however, the chest compression reaction times were similar between the first and second simulations (table 6).

During the first simulations, chest compression reaction times in method A groups were longer than those of method B groups (method A vs $\mathrm{B}=64.6 \pm 53.6$ vs 28.8 $\pm 11.6 \mathrm{~s}, \mathrm{p}=0.008)$. There were no differences between method $\mathrm{A}$ and $\mathrm{B}$ chest compression reaction times of the second simulations. Method A groups had shorter chest compression reaction times between the first and second simulations than method B groups (method A vs $\mathrm{B}=-41.3 \pm 54.9$ vs $-2.5 \pm 19.9 \mathrm{~s}, \mathrm{p}=0.008$; table 7 ).

\section{DISCUSSION}

The major findings of our study are: (1) leadership, teamwork and team member skills were improved after training; (2) in the first CPR simulation, an initial movie 
Table 6 Comparing the reaction times of first simulation and second simulation in all groups, method A and B groups

\begin{tabular}{lccc}
\hline & First simulation & Second simulation & p Value \\
\hline All groups & & & \\
Chest compression (s) & $43.3 \pm 38.8$ & $25.1 \pm 16.4$ & 0.020 \\
Defibrillation (s) & $178.2 \pm 67.7$ & $166.1 \pm 73.9$ & 0.507 \\
Method A groups & & & 0.019 \\
$\quad$ Chest compression (s) & $64.6 \pm 53.6$ & $23.3 \pm 8.9$ & 0.636 \\
Defibrillation (s) & $182.8 \pm 77.8$ & $167.2 \pm 74.2$ & 0.588 \\
Method B groups & & $26.3 \pm 20.1$ & 0.660 \\
$\quad$ Chest compression (s) & $28.8 \pm 11.6$ & $165.3 \pm 75.7$ & \\
Defibrillation (s) & $175.0 \pm 61.9$ & & \\
\hline
\end{tabular}

Table 7 Comparing the reaction times in method $A$ and $B$ groups

\begin{tabular}{lccc}
\hline & Method A & Method B & p Value \\
\hline Reaction times of first simulation & & & \\
$\quad$ Chest compression (s) & $64.6 \pm 53.6$ & $28.8 \pm 11.6$ & 0.008 \\
$\quad$ Defibrillation (s) & $182.8 \pm 77.8$ & $175.0 \pm 61.9$ & 0.766 \\
Reaction times of second simulation & & & 0.577 \\
$\quad$ Chest compression (s) & $23.3 \pm 8.9$ & $26.3 \pm 20.1$ & 0.946 \\
$\quad$ Defibrillation (s) & $167.2 \pm 74.2$ & $165.3 \pm 75.7$ & 0.008 \\
Changes of reaction times between first and second simulation & & 0.880 \\
$\quad$ Chest compression (s) & $-41.3 \pm 54.9$ & $-2.5 \pm 19.9$ & $-9.7 \pm 94.2$ \\
$\quad$ Defibrillation (s) & $-15.6 \pm 116.1$ & & \\
\hline
\end{tabular}

did not produce greater improvement in leadership, teamwork and team member skills; (3) in the second CPR simulation, method A's video exposure followed by first CPR simulation further increased student leadership skills compared with a method B's first CPR simulation followed by video exposure.

\section{Training improves leadership, teamwork and team member skills}

Our study shows that overall, students' leadership, teamwork and team member skills can be improved after CPR training. The finding is consistent with the meta-analysis that technology-enhanced simulation training is effective in health professions education. ${ }^{7}$ Medical student training and experience in cardiac arrest situations is limited. One study showed that ACLS training with high-fidelity simulations significantly improved knowledge and psychomotor skills in medical students. ${ }^{8}$ In another study comparing CPR teams who received additional leadership instruction versus those who received additional technical instruction, teams of medical students who received leadership instruction had significantly greater resuscitation outcomes and performance..$^{9}$ In a similar study comparing CPR performance of teams of medical students whose leaders either received team leader training or additional advanced life support training, those with leadership-trained individuals had greater protocol adherence scores as well as leadership behaviour. ${ }^{10}$ Furthermore, after all the teams were given technical versus leadership instructions, students in leadership-trained teams showed greater overall CPR performance for up to 4 months. ${ }^{9}$
Our study found that video showing and a first CPR simulation can improve students' second CPR simulation skills in organisation, order delivery, teammate support and awareness in the leadership category; communication, cooperation, experience sharing, ACLS adherence and task completion in the teamwork category; and effective/correct patient contact, chest compression and IV medication in the team member skills category. This positive effect may be due to multiple combinatorial factors. Since the main concepts of the video included elements of team dynamics, team leader traits and team member traits, the first factor may be an enhanced understanding of concepts learnt in the video through either concept implementation during a subsequent first CPR simulation (method A) or concept analysis after a prior first CPR simulation (method B). Second, subsequent or prior simulation practice during a first CPR simulation allows students to gain procedural experience and familiarity with the simulation environment and resuscitation equipment. Finally, following the first CPR simulation, debriefing with an attending physician before the second CPR simulation may also serve to solidify student understandings of how video concepts may be integrated into the resuscitation protocol.

\section{An initial movie did not improve leadership, teamwork} and team member skills

Initial movie showings prior to a first CPR simulation (method A) did not improve leadership, teamwork or team member skills significantly more than first CPR simulation taken without initial movie showings 
(method B). In our study, first CPR simulation scores in checklist rating scores of leadership, teamwork and team member skills categories as well as global rating scores of students taught using method A did not differ significantly from first CPR simulation scores of those taught using method $\mathrm{B}$. This indicates that a movie alone may be insufficient to increase student's CPR performance in leadership, teamwork and team member skills.

Although multimedia tools for learning CPR skill in medical education have shown to be beneficial, the results were not consistent in all studies. In one study comparing basic life support (BLS) education using videos versus pictures, results indicated that pictures were a more effective means to increase BLS skills. ${ }^{19}$ A suggested hypothesis was that animations are often 'too complex or too fast to be accurately perceived'. A further study could compare simulation competency of students who viewed pictures detailing CPR procedures and teamwork skill concepts versus those who watched didactic videos demonstrating CPR procedures and skills.

\section{The chronological order of video exposure and first CPR} simulation affects leadership skills and performance

Using method A, in which an initial movie showing was followed by a first CPR simulation, increased leadership test performance (specifically in the support subcategory) more than method $\mathrm{B}$, in which movie showing followed an initial first CPR simulation. This leadership enhancement may be due to a heightened understanding of team dynamic, membership and leadership concepts learnt in the initial didactic video and subsequent implementation of these concepts in an interactive simulation setting during the first CPR simulation. Following the first CPR simulation, debriefing with an attending physician before the second CPR simulation may also serve to solidify student's understanding of how video concepts may be integrated into the resuscitation protocol.

\section{Improvement of chest compression reaction times during} first CPR simulations versus second CPR simulations

Overall, the reaction times of chest compressions in the second simulations were significantly shorter than those of the first simulations. Although in method A groups chest compression reaction times of the first simulation were longer than those of method B groups, the second simulation chest compression reaction times of the two groups were similar. Student reaction times to defibrillation did not vary significantly in first CPR simulationsecond CPR simulation and cross-method comparisons. Since chest compression reaction times of the first simulation were different in the two groups, it cannot be concluded that method A groups had more significant improvement. One study also showed that reaction times for CPR and defibrillation of medical students groups conducting passive observation of CPR scenarios did not differ with those of groups conducting active observation with checklists. ${ }^{20}$ Although checklist results indicated overall improvement of student scores after video exposure combined with a first CPR simulation, reaction times did not change significantly, perhaps showing that reaction times do not represent leadership and teamwork skill improvement.

\section{Study limitations}

Our study has some limitations. The first is the lack of a control group who never watched the video or who did not engage in a first CPR simulation. Owing to the ethical prioritisation of student learning experiences, all student groups received a first CPR simulation and video showing, though in varying chronological orders. Second, study results must be considered with acknowledgement of a relatively small sample size. A further study is needed to verify these findings with a larger sample. Third, the study was designed only observationally and we did not match the baseline characteristics of the participants in method A and B groups. However, the baseline characteristics of the participants in the two groups were similar, including gender, degree, experience on resuscitation and student numbers in each team. Fourth, the evaluation process included real-time evaluation by one attending physician who taught the students and video-recording evaluation by two independent individuals. There may be bias in the real-time evaluation by only one attending physician. However, we compared the attending physician's rating scores for 40 simulation rounds with that of one nursing practitioner using the same evaluation form in the preliminary status of the study and found that the rating scores were similar among the two raters (see online supplementary appendix 2). Future studies should be conducted with two independent assessors. ${ }^{18}$ Finally, our CPR video was from the year 2005. During the resuscitation training, we followed the updated CPR guidelines. Although the CPR video was old, the main concepts of the video included elements of team dynamics, team leader traits and team member traits, which were still pertinent.

\section{CONCLUSION}

Although both the teaching strategies improved leadership, teamwork and team member performance, method A's video exposure followed by first CPR simulation further increased student leadership skills compared with a method B's first CPR simulation followed by video exposure. This finding supports that initial video instruction followed by CPR simulation is a better strategy for leadership skills training. However, the study was limited due to its observational design and small sample size. Further studies are still necessary to clarify our findings.

\section{Author affiliations}

${ }^{1}$ Department of Medical Education, Taipei Veterans General Hospital, Taipei, Taiwan

${ }^{2}$ Western University of Health Sciences, College of Osteopathic Medicine of the Pacific, Pomona, California, USA 
${ }^{3}$ Division of Cardiology, Department of Medicine, Taipei Veterans General Hospital, Taipei, Taiwan

${ }^{4}$ Cardiovascular Research Center, National Yang-Ming University, Taipei, Taiwan

${ }^{5}$ Institute of Pharmacology, National Yang-Ming University, Taipei, Taiwan

${ }^{6}$ Department of Medical Research, Taipei Veterans General Hospital, Taipei,

Taiwan

${ }^{7}$ Institute of Clinical Medicine, National Yang-Ming University, Taipei, Taiwan

Acknowledgements CW currently attends the College of Osteopathic Medicine of the Pacific, Western University of Health Sciences in Pomona CA. At the time this research was conducted, she was solely affiliated with the Taipei Veterans General Hospital.

Contributors $\mathrm{C}-\mathrm{CH}$ was the principal investigator in the study and contributed to the study conception and design, implementation, statistical analysis, interpretation, and preparation of the manuscript. CW contributed to the study conception and design, and preparation of the manuscript. S-JL contributed to the study conception and design, and preparation of the manuscript. J-WC contributed to the study conception and design, statistical interpretation, and preparation of the manuscript. All authors have approved the final manuscript for publication and agree to be accountable for all aspects of the work.

Funding This work has been supported by research grants V98S1-020, V99S1-007, V100EA-024, V101EA-013, V102EA-018 and V104EA-008 from Taipei Veterans General Hospital, Taipei, Taiwan, R.O.C., by research grants NSC 100-2314-B-075-055 and NSC 102-2314-B-075-022-MY3 from Taiwan's National Science Council, Taiwan, R.O.C., and by research grant MOST 105-2511-S-075-004 from Ministry of Science and Technology, Taiwan, R.O.C.

Competing interests None declared.

Provenance and peer review Not commissioned; externally peer reviewed.

Data sharing statement No additional data are available.

Open Access This is an Open Access article distributed in accordance with the Creative Commons Attribution Non Commercial (CC BY-NC 4.0) license, which permits others to distribute, remix, adapt, build upon this work noncommercially, and license their derivative works on different terms, provided the original work is properly cited and the use is non-commercial. See: http:// creativecommons.org/licenses/by-nc/4.0/

\section{REFERENCES}

1. Hasselqvist-Ax I, Riva G, Herlitz J, et al. Early cardiopulmonary resuscitation in out-of-hospital cardiac arrest. $N$ Engl J Med 2015;372:2307-15.

2. Talikowska M, Tohira H, Finn J. Cardiopulmonary resuscitation quality and patient survival outcome in cardiac arrest: a systematic review and meta-analysis. Resuscitation 2015;96:66-77.

3. McEvoy MD, Field LC, Moore HE, et al. The effect of adherence to ACLS protocols on survival of event in the setting of in-hospital cardiac arrest. Resuscitation 2014;85:82-7.
4. Yannopoulos D, Aufderheide TP, Abella BS, et al. Quality of CPR: an important effect modifier in cardiac arrest clinical outcomes and intervention effectiveness trials. Resuscitation 2015;94:106-13.

5. Abella BS, Alvarado JP, Myklebust $\mathrm{H}$, et al. Quality of cardiopulmonary resuscitation during in-hospital cardiac arrest. JAMA 2005;293:305-10.

6. Graham R, McCoy MA, Schultz AM, Committee on the Treatment of Cardiac Arrest: Current Status and Future Directions; Board on Health Sciences Policy; Institute of Medicine, eds. Strategies to improve cardiac arrest survival: a time to act. Washington DC: National Academies Press (US), 2015.

7. Cook DA, Hatala R, Brydges R, et al. Technology-enhanced simulation for health professions education: a systematic review and meta-analysis. JAMA 2011;306:978-88.

8. Langdorf MI, Strom SL, Yang L, et al. High-fidelity simulation enhances ACLS training. Teach Learn Med 2014;26:266-73.

9. Hunziker S, Bühlmann C, Tschan F, et al. Brief leadership instructions improve cardiopulmonary resuscitation in a high-fidelity simulation: a randomized controlled trial. Crit Care Med 2010;38:1086-91.

10. Fernandez CE, Boos M, Ringer C, et al. Effect of CRM team leader training on team performance and leadership behavior in simulated cardiac arrest scenarios: a prospective, randomized, controlled study. BMC Med Educ 2015;15:116.

11. Andersen $P O$, Jensen MK, Lippert A, et al. Identifying non-technical skills and barriers for improvement of teamwork in cardiac arrest teams. Resuscitation 2010;81:695-702.

12. Deakin CD, Nolan JP, Soar J, et al. European Resuscitation Council Guidelines for Resuscitation 2010 Section 4. Adult advanced life support. Resuscitation 2010;81:1305-52.

13. Bhanji F, Mancini ME, Sinz E, et al. Part 16: education, implementation, and teams: 2010 American Heart Association Guidelines for Cardiopulmonary Resuscitation and Emergency Cardiovascular Care. Circulation 2010;122(Suppl 3):S920-33.

14. Hunziker S, Johansson AC, Tschan F, et al. Teamwork and leadership in cardiopulmonary resuscitation. J Am Coll Cardiol 2011:57:2381-8.

15. Cheng A, Lockey A, Bhanji F, et al. The use of high-fidelity manikins for advanced life support training-A systematic review and meta-analysis. Resuscitation 2015;93:142-9.

16. Beskind DL, Stolz U, Thiede R, et al. Viewing a brief chest-compression-only CPR video improves bystander CPR performance and responsiveness in high school students: a cluster randomized trial. Resuscitation 2016;104:28-33.

17. Adams AJ, Wasson EA, Admire JR, et al. A comparison of teaching modalities and fidelity of simulation levels in teaching resuscitation scenarios. J Surg Educ 2015;72:778-85.

18. Husebø SE, Bjørshol CA, Rystedt $\mathrm{H}$, et al. A comparative study of defibrillation and cardiopulmonary resuscitation performance during simulated cardiac arrest in nursing student teams. Scand J Trauma Resusc Emerg Med 2012;20:23.

19. Iserbyt $P$, Charlier N, Mols L. Learning basic life support (BLS) with tablet PCs in reciprocal learning at school: are videos superior to pictures? A randomized controlled trial. Resuscitation 2014;85:809-13.

20. Dilley SJ, Weiland TJ, O'Brien R, et al. Use of a checklist during observation of a simulated cardiac arrest scenario does not improve time to CPR and defibrillation over observation alone for subsequent scenarios. Teach Learn Med 2015;27:71-9. 